\title{
Study on the Third-order Nonlinear Optical Properties of bis(tetraethylammonium) bis(1,3-dithiole-2-thione-4,5-dithiolato)cadium
}

\author{
Hongliang Yang ${ }^{1}$, Fujun Zhang ${ }^{1}$, Xinqiang Wang ${ }^{2}$, Guanghui Zhang ${ }^{2}$ \\ ${ }^{1}$ Department of Optics, Shandong University, Jinan,250100,China \\ ${ }^{2}$ State Key Laboratory of Crystal, Shandong University, Jinan,250100,China \\ Email: yanghongliang@sdu.edu.cn, xqwang@sdu.edu.cn
}

Received 2013

\begin{abstract}
A dmit ${ }^{2-}$ salt: bis(tetraethylammonium)bis(1,3-dithiole-2-thione-4,5-dithiolato)cadium (CADMIT) was synthesized. The Optical Kerr Effect (OKE) signal of its acetonitrile solution was measured by femtosecond optical Kerr gate technique. Using $\mathrm{CS}_{2} \mathrm{OKE}$ signal as reference signal measured under identical conditions, the third-order optical nonlinear susceptibility, $\chi^{(3)}$, of the sample solution was obtained to be about $2.98 \times 10^{-14}$ esu at the concentration of $1.57 \times 10^{-3} \mathrm{M}$. The second-order hyperpolarizability of its molecular was estimated to be as large as $1.23 \times 10^{-32}$ esu. Its response time was about $195 \mathrm{fs}$, which is believed to be the contribution from the delocalized electrons.
\end{abstract}

Keywords: Optical Nonlinearity; Kerr Gate Technology; Response Time

\section{Introduction}

Recently, nonlinear optical materials have attracted much interest because of their potential in fabricating ultrafast optical switching and processing devices [1-3]. For this purpose, many materials, including semiconductors, polymers, nanomaterials and inorganic materials, have been studied. Among them, $\pi$-conjugated organic material has been paid great attention for its high nonlinear optical (NLO) properties and ultrafast response [4]. Especially, the organometallic and coordination material has been attracting great concern, because it has the advantages of architectural flexibility, ease of fabrication and tailoring, and high NLO properties of organics. Simultaneously, it possesses good transmissivity, temporal and thermal stability of inorganic matter. Such material also has strongly enhanced second-order hyperpolarizability by introducing a metal atom.

Currently, the synthesis and characterization of 1,3dithiole-2-thione-4,5-dithiolate (dmit) complexes and related selenlum- and oxygen-substituted isologs have been studied. As a special $\pi$-electron delocalization conjugated system, dmit and related ligand complexes have been used in the assembly of highly electrically conducting radical anion salts and charge-transfer complexes. They are generally used as important building blocks for organic, organometallic and coordination complex electrical conductors and superconductors. The $\pi$-electron delocalization in conjugated systems can also contribute to the ultrafast response capability and large third-order nonlinearity. Recently, some of these complexes possessing good second-order[5,6] and third-order[7-11] NLO properties have been reported. In this paper, a dmit $^{2-}$ salt: bis(tetraethylammonium)bis(1,3-dithiole-2thione-4,5-dithiolato)cadium was synthesized. The Optical Kerr Effect (OKE) signal of its acetonitrile solution was measured by femtosecond optical Kerr gate technique.

\section{Material Preparation and Experiment Method}

The preparation method of CADMIT crystal was a modification according to literature method [12]. The sample solution was prepared using acetonitrile as the solvent whose concentration is $1.57 \times 10^{-3} \mathrm{M}$.

The femtosecond optical Kerr gate technique was applied in this experiment whose measurement setup is illustrated in Figure 1. The light source, centered at 800 $\mathrm{nm}$, is a Ti:sapphire femtosecond laser system (Mira 900F, Coherent, USA), which is pumped by a multiline $\mathrm{Ar}^{+}$laser system (Innova 400, Coherent, USA) at $11 \mathrm{~W}$. The repetition rate is $76 \mathrm{MHz}$ and its pulse width is 120 fs. The femtosecond laser beam whose average power was about $0.64 \mathrm{~W}$ is split into a probe beam and a pump beam by a beam splitter. The intensity ratio for the probe beam and the pump beam is $1: 10$. The polarization of the probe beam is carefully adjusted at $45^{\circ}$ to the linear polar- 
ized pump beam. The pump beam passes through a delay line driven by a step motor. Then it is carefully reflected parallel to the probe beam at an adjacent configuration. The two beams are focused by a convex lens. At the focus of the lens, the two beams overlap each other. This focus would be enclosed within the sample cell of $1 \mathrm{~mm}$ in thickness during the measurement. After transmitting through the sample cell, the pump beam is blocked, while the probe one passes an analyzer, of which the transmission axis is strictly perpendicular to that of the probe beam. Finally, the OKE signal was recorded as a function of pump-probe delay by an amplified photodiode and recorded by a digital lock-in amplifier (SR830, Stanford, USA) referenced to the chopper frequency. Both delay stage and lock-in amplifier were controlled by a personal computer.

\section{Results and Discussions}

The molecular structure of CADMIT is illustrated in Figure 2 and the absorption spectrum of its acetonitrile solution is in Figure 3. Its absorption coefficient at 800 $\mathrm{nm}$ is very small and suggest that $800 \mathrm{~nm}$ is far from its resonant band. And the absorption around $400 \mathrm{~nm}$ is also not srong which indicates its two-photon absorption at $800 \mathrm{~nm}$ has small effect on our measurement result. In the OKE experiment, $\mathrm{CS}_{2}$ was used as reference. Since the sample is prepared as a solution, the measured thirdorder NLO susceptibility, $\chi^{(3)}$, was the combination of the response from both the sample solute and solvent. Therefore in the experiment, we measured the OKE signals of $\mathrm{CS}_{2}$, the solvent, and sample solution, consecutively. With the solvent signal was removed from the sample solution signal, we obtained the pure signal of sample. Then we used the following equation to calculate its third-order NLO susceptibility $\chi^{(3)}$ [13-15].

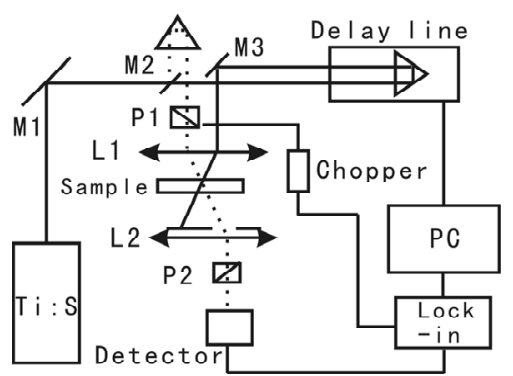

Figure 1. Experimental Schematic drawing for femtosecond Optical Kerr Effect.

$$
\left(\left(\mathrm{C}_{2} \mathrm{H}_{5}\right)_{4} \mathrm{~N}\right)_{2}\left[\mathrm{~S}=\left.\int_{\mathrm{S}}^{\mathrm{S}}\right|_{\mathrm{S}} ^{\mathrm{S}} \mathrm{Cd} \|_{\mathrm{S}}^{\mathrm{S}}=\mathrm{S}\right]
$$

Figure 2. Molecular structure of CADMIT.

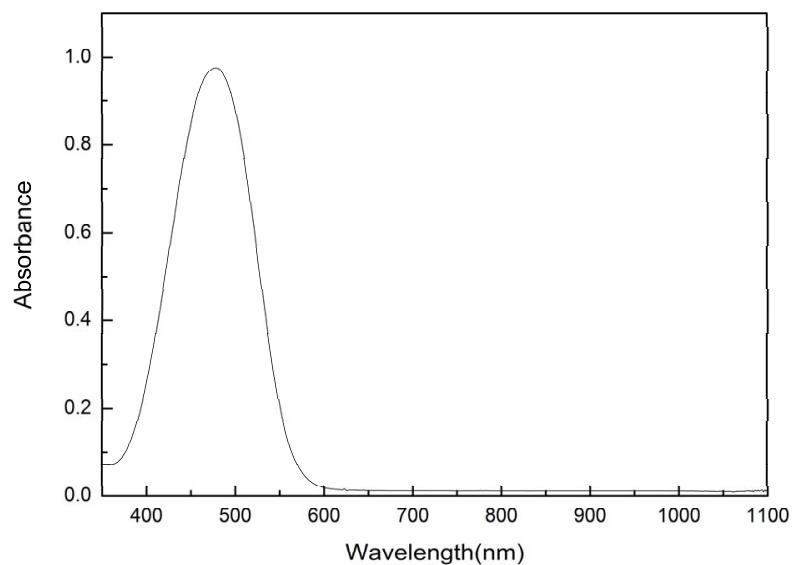

Figure 3. Absorption spectrum of acetonitrile solution of CADMIT.

$$
\chi_{\mathrm{S}}{ }^{(3)}=\chi_{R}{ }^{(3)}\left(\frac{I_{S}}{I_{R}}\right)^{1 / 2}\left(\frac{n_{S}}{n_{R}}\right)^{2}
$$

The subscripts $\mathrm{S}$ and $\mathrm{R}$ represent the sample of CADMIT and reference sample $\mathrm{CS}_{2}$. I indicates the intensity of OKE and $n$ is the refractive index. The concentration of the sample is very low in the solvent, so we use the refractive index of acetonitrile as the index of solution, which is 1.34. The $\mathrm{n}$ for $\mathrm{CS}_{2}$ is 1.62 . The third order nonlinear susceptibility of $\mathrm{CS}_{2}$ is estimated to be $1 \times$ $10^{-13}$ esu in femtosecond time scale [16]. Using the equation, we can get the third-order NLO susceptibility $\chi^{(3)}$ of sample directly by measuring the OKE signal intensity of both sample and reference under identical conditions.

The OKE signal of the sample solution was measured and illustrated in Figure 4, in which the small contribution from the solvent was subtracted. Using Eq. (1) and the measured signals of $\mathrm{CS}_{2}$ and sample solution, the third-order susceptibility $\chi^{(3)}$ of sample solution was obtained to be $2.98 \times 10^{-14}$ esu at the concentration of 1.57 $\times 10^{-3} \mathrm{M}$. The second-order hyperpolarizability, $\gamma$, of the sample molecule may be estimated through the equation $[17,18]$

$$
\gamma=\chi^{(3)} /(N L)
$$

where $N$ is the concentration of the solution and $\mathrm{L}$ is the local field correction factor which is defined as $\left[\left(n^{2}+2\right) / 3\right]^{4}$ ( $\mathrm{n}$ is the refractive index of solution). By Eq. (2), we may calculate $\gamma$ of CADMIT as $1.23 \times 10^{-32}$ esu using the measured $\chi^{(3)}=2.98 \times 10^{-14}$ esu.

From Figure 4, we obtained the response time of bis(tetraethylammonium)bis(1,3-dithiole-2-thione-4,5-dit hiolato)cadium to be about 195 fs, which is commonly accepted to be the contribution from the transient motion of the $\pi$-conjugated-electron distribution. Normally, the time domain response of the sample should contain several processes of different time scales. Using the femtosecond OKE measurement system, some subpicosecond 


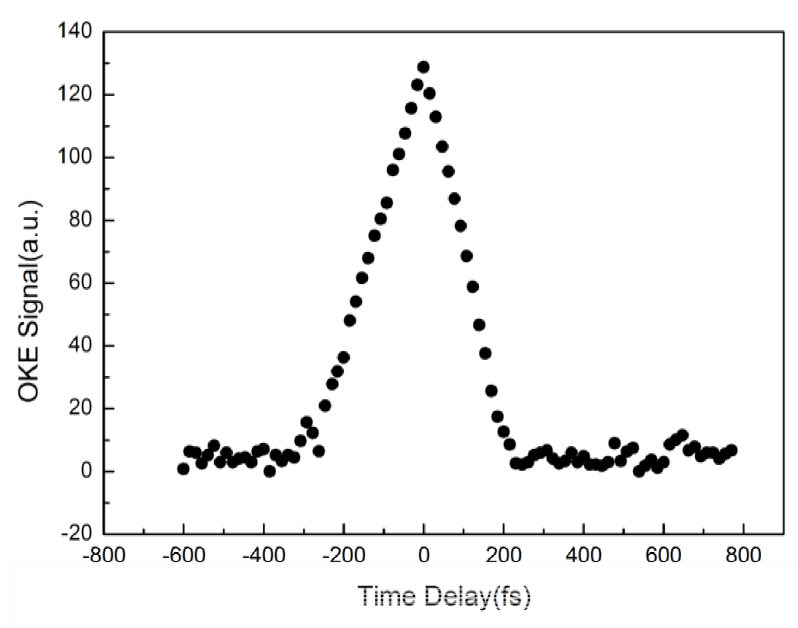

Figure 4. OKE signals of CADMIT.

processes can be distinguished. The third-order NLO response time induced by delocalized electrons is believed to be $10^{-14}-10^{-15} \mathrm{~s}$. While the response time induced by molecular reorientation is $10^{-11}-10^{-12} \mathrm{~s}$ and that induced by density change is $10^{-8}-10^{-9} \mathrm{~s}$.

\section{Conclusions}

CADMIT was synthesized by making some modifications to the literature method. The absorption spectrum of its acetonitrile solution was measured, which shows that the salt has good transmittance from $600 \mathrm{~nm}$ to infrared band. Its third-order optical nonlinearity $\chi^{(3)}$ was studied by a transient OKE measurement and was about $2.98 \times$ $10^{-14}$ esu at the concentration of $1.57 \times 10^{-3} \mathrm{M}$. Its thirdorder optical nonlinearity response time was obtained and be about $195 \mathrm{fs}$, which is commonly accepted to be the contribution from the transient motion of the $\pi$-conjugated-electron distribution. Its second-order hyperpolarizability, as large as $1.23 \times 10^{-32}$ esu, was estimated. These features indicate CADMIT is a potential photonics material in future.

\section{Acknowledgements}

The authors acknowledge the financial support of the National Natural Science Foundation (Grant No. 1100 4123).

\section{REFERENCES}

[1] C. Halvorson, A. Hays, B. Kraabel, R. Wu, F. Wudl and A. J. Heeger, "A 160-Femtosecond Optical Image Processor Based on a Conjugated Polymer,” Science, Vol. 265, 1994,pp. 1215-1216. doi:10.1126/science.265.5176.1215

[2] S. R. Marder, W. E. Torruellas, M. B. Desce, V. Ricci, G. I. Stegeman, S. Gilmour, T. L. Bredas, J. Li, G. U. Bub- litz and S. G. Boxer, "Large Molecular Third-Order Optical Nonlinearities in Polarized Carotenoids," Science, Vol. 276, 1997, pp. 1233-1236. doi:10.1126/science.276.5316.1233

[3] A. P. Slepkov, F. A. Hegmann, Y. M. Zhao, et al., "Ultrafast Optical Kerr Effect Measurements of Third- Order Nonlinearitiesin Cross-Conjugated Iso-Polydiacetylene Oligomers,” Journal of Chemical Physics, Vol. 116, 2002, pp. 3834-3840. doi:10.1063/1.1447908

[4] A. S. L. Gomes, L. Demenicis, D. V. Petrov, et al., "Time-Resolved Picosecond Optical Nonlinearity and All- Optical Kerr Gate in Poly (3-Hexadecylthiophene)," Applied Physics Letters, Vol. 69, 1996, pp. 2166-2168.

[5] Q. Fang, M. H. Jiang, Z. Qu, J. H. Cai, H. Lei, W. T. Yu, and Z. Zhuo, "Non-Linear Optical Properties of DMIT Derivatives,” Journal of Materials Chemistry, Vol. 4, 1994, pp. 1041-1045. doi:10.1039/jm9940401041

[6] J. Zhai, C.H. Huang, T.X. Wei, L.B. Gan and H. Cao, "The Photoelectric Conversion and Second Harmonic Generation Properties of the Transition Metal- Containing Coplexes[(CH3)2NC6H4-CH=CH-C5H4N-C18H37]2M( dmit)2(M=Cd,Ni),’Polyhdron,Vol.18,pp.1513-1518,1999. doi:10.1016/S0277-5387(99)00020-0

[7] C. S. Winter, S. N. Oliver, R. J. Manning, J. D. Rush, et al., "Non-Linear Optical Studies of Nickel Dithiolene Complexes," Journal of Materials Chemistry, Vol. 2, 1992, pp. 443-447. doi:10.1039/jm9920200443

[8] J. L. Zuo, T. M. Yao, F. You, X. Z. You, H. K. Fun and B. C. Yip, "Syntheses, Characterization and Non-Linear Optical Properties of Nickel Complexes of Multi-Sulfur 1,2-Dithioiene with Strong Near-IR Absorption,” Journal of Materials Chemistry, Vol. 6, 1996, pp. 1633-1637. doi:10.1039/jm9960601633

[9] J. F. Bai, J. L. Zuo, W. L. Tan, W. Ji, Z. Shen, H. K. Fun, et al., "Synthesis, Structure and Optical Limiting Effect of Two Newnickel Complexes Containing Strongly Bound Geometrically Fixedmulti-Sul-Fur 1,2-Dithiolene Ligands Showing Remarkable Near-IRab-Sorption," Journal of Materials Chemistry, Vol. 9, 1999, pp. 2419-2423. doi:10.1039/a902295c

[10] J. Dai, G. Q. Bian, X. Wang, Q. F. Xu, M. Y. Zhou, M. Munakata, et al., "A New Method to Synthesize Unsymmetrical Dithiolene Metal Complexes of 1,3-Dithiole-2-Thione-4,5-Dithiolate for Third-Order Nonlinear Optical Applications,” Journal of America Chemistry Society, Vol. 122, 2000, pp. 11007-11008. doi:10.1021/ja001976e

[11] C. M. Liu, D. Q. Zhang, Y. L. Song, C. L. Zhan, Y. L. Li and D. B. Zhu, "Synthesis, Crystal Structure and Third-Order Nonlinear Optical Behavior of a Novel Dimeric Mixed-Ligand Zinc(II) Complex of 1,3-Dithiole-2-Thione-4,5-Dithiolate,” European Journal of Inorganic Chemistry, Vol. 2002, 2002, pp. 1591-1594. doi:10.1002/1099-0682(200207)2002:7<1591::AID -EJIC1591>3.0.CO;2-T

[12] C. S. Wang, A. S. Batsanov, M. R. Bryce and J. A. K. Howard, “An Improved Large-Scale (90 g) Synthesis of 
Bis(tetraethylammnium)Bis(1,3-Dithiole-2-Thione-4,5-Di thiol)zincate:Synthesis and X-ray Crystal Structures of Bicyclic and Tricyclic 1,4-Dithiocines Derived from 1,3-Dithiole-2-thione-4,5-dithiolate(DMIT),"Synthesis, Vo l.1998, pp. 1615-1618. doi:10.1055/s-1998-2197

[13] B. K. Mandal, B. Bihari, A. K. Sinha, M. Kamath and L. Chen, "Third-Order Nonlinear Optical Response in A Multilayered Phthalocyanine Composite,” Applied Physics Letters, Vol. 66, 1995, pp. 932-934. doi:10.1063/1.113601

[14] S. F. Wang, W. T. Huang, T. Q. Zhang, H. Yang, et al., "Third-Order Nonlinear Optical Properties of Didodecyldimethylammonium-Au(dmit) $)_{2}$,"Applied Physics Letters, Vol. 75,1999, pp. 1845-1847,.

[15] D. J. Mcgraw, A. E. Siegman, G. M. Wallraff, and R. D. Millar, "Resolution of the Nuclear and Electronic Contributions to the Optical Nonlinearity in Polysilanes," Applied Physics Letters, Vol. 54, 1989, pp. 1713-1715. doi:10.1063/1.101290

[16] K. Minoshima, M. Taiji and T. Kobayashi, "Femtosecond Time-Resolved Interferometry for the Determinatio of Complex Nonlinear Susceptibility,” Optics Letters, Vol. 16, 1991, pp. 1683-1685. doi:10.1364/OL.16.001683

[17] Q. H. Gong, Y. X. Sun, Z. J. Xia, et al., "Nonresonant Third-Order Optical Nonlinearity of All-Carbon MoleculesC $_{60}$,"Journal of Applied Phyics, Vol. 71, 1992, pp. 3025-3026. doi:10.1063/1.351391

[18] M. T. Zhao, B. P. Singh and P. N. Prasad, "A Systematic Study of Polarizability and Microscopic Third - Order Optical Nonlinearity in Thiophene Oligomers,” Journal of Chemical Physics, Vol. 89, 1988, pp. 5535-5541. doi:10.1063/1.455560 